\title{
DESENVOLVIMENTO DE UM PRODUTO INOVADOR COM PLANEJAMENTO, CRIATIVIDADE E QUALIDADE: O CASO DO PORTA ESMALTE
}

\author{
Annabel Barra Pinto (UFERSA, Brasil) bebellbarra@hotmail.com \\ Raimundo Alberto Rego Junior (UFERSA, Brasil) albertojuniorpdf@hotmail.com \\ Rochelly Sirremes Pinto (UFERSA, Brasil) rochellykarillo@hotmail.com \\ Romario Allysson Dantas de Sousa Camara (UFERSA, Brasil) romarioallysson@yahoo.com.br \\ Maria Aridenise Macena Fontenelle (UFERSA) aridenise@ufersa.edu.br
}

Resumo: A competitividade atual, a melhoria continua e a velocidade das informações obriga as empresas a melhorar seus procedimentos organizacionais. A vantagem competitiva está diretamente relacionada à sua capacidade de introduzir no mercado novos produtos e serviços, com conteúdo tecnológico e características de qualidade, desempenho, custo e distribuição que satisfaçam as exigências dos consumidores. Na literatura são propostas diversas metodologias para o desenvolvimento de novos produtos, cabendo às empresas encontrar ou adequar àquela que melhor se adapte a sua realidade $\mathrm{e}$ cultura. Baseado nisso, este trabalho propõe a aplicação da metodologia proposta por diferentes autores no processo de desenvolvimento de um produto de baixa complexidade, como uma experiência didática vivida por um grupo de aluno da disciplina de Projeto de Produto do curso de engenharia de produção.

Palavras-chave: Projeto de produto. Desenvolvimento de produto. Metodologia de projeto.

Abstract: The current competitiveness, continuous improvement and the speed of information forces companies to improve their organizational procedures. The competitive advantage is directly related to its ability to market new products and services, technological content and quality features, performance, cost and distribution that meet consumer demands. In the literature several methodologies proposed for the development of new products, leaving companies to find or adjust to what best suits your reality and culture. Based on this, this paper proposes the application of the methodology proposed by different authors in the development of a product with low complexity process as a learning experience lived by a group of student discipline Product Design course in production engineering.

keyword: Product design. Product development. Design methodology. 


\section{Introdução}

As constantes mudanças no âmbito econômico-financeiro aliadas a globalização têm modificado fortemente o contexto competitivo das organizações de modo que a permanência destas está condicionada à satisfação dos seus clientes e ao posicionamento adequado no mercado frente às concorrentes. Atrelado a isso existe a necessidade constante de desenvolver produtos cada vez mais diferenciados, seja na qualidade, na inovação ou tecnologia.

Mediante a isso o desenvolvimento de produtos surge como uma necessidade. A capacidade de desenvolver e introduzir no mercado novos produtos proporciona maior lucratividade, crescimento econômico e vantagens competitivas.

Desenvolver produtos consiste em um conjunto de atividades por meio das quais se busca, a partir das necessidades do mercado e das possibilidades e restrições tecnológicas, e considerando as estratégias competitivas e de produto da empresa, chegar às especificações de projeto de um produto e de seu processo de produção, para que a manufatura seja capaz de produzi-lo (ROZENFELD, 2006).

Ainda segundo Rozenfeld (2006) um novo produto necessita de um projeto bem segmentado que descreva ordenadamente todas as fases de planejamento e execução, além do mapeamento das necessidades e os desejos dos clientes.

Neste contexto, o presente trabalho tem como propósito o de detalhar as fases do processo de desenvolvimento de um produto por meio de uma aplicação prática na construção de um porta-esmalte, com base nas etapas de uma metodologia específica, proposta e fundamentada nas referenciadas na literatura.

\section{Revisão literária}

A manufatura e o aperfeiçoamento de produtos não é uma atividade recente. Segundo Romeiro (2009) a relação do homem com os produtos tridimensionais é tão antiga quanto à própria humanidade e adveio da necessidade de manter-se vivo. Os produtos facilitam a vida do homem e tendem a evoluir com ele.

Um produto é algo que pode ser oferecido a um mercado para satisfazer uma necessidade ou desejo (KOTLER, 2000, p. 416). Já de acordo com Kaminski (2000) o produto é o resultado de um projeto de engenharia, desenvolvido e disponibilizado aos clientes a fim de satisfazer necessidades individuais e coletivas.

Projetar novos produtos e lançá-los ao mercado rapidamente é o desafio enfrentado pelos fabricantes nos mais diversos setores (CHASE, 2006). E devido a isso, os esforços em superar cada vez mais as expectativas dos clientes estão ainda maiores, seja na produção de bens ou serviços.

As empresas estão empenhadas em buscar cada vez mais desenvolver um produto que supra todas as exigências do mercado. A partir daí o Processo de desenvolvimento de produtos (PDP) surge como um método fundamental para as empresas manterem-se atuantes e competitivas. 
Diversos autores compartilham da idéia de que a adoção de um método específico de PDP torna-se primordial na elaboração de um novo produto. Existem na literatura diferentes modelos e propostas de autores acerca das etapas de desenvolvimento. A Figura 1 mostra os diferentes modelos propostos por alguns dos principais pesquisadores encontrados no aporte teórico sobre o assunto.

FIGURA 1: DIFERENTES METODOLOGIAS DE PDP

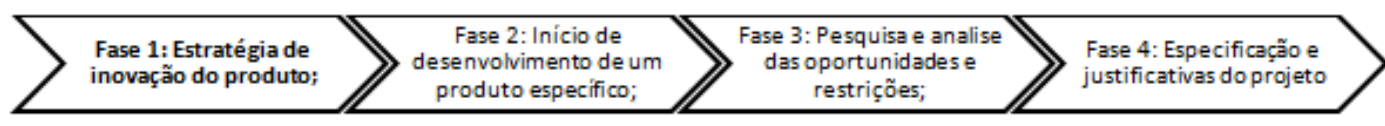

Baxter (2003)

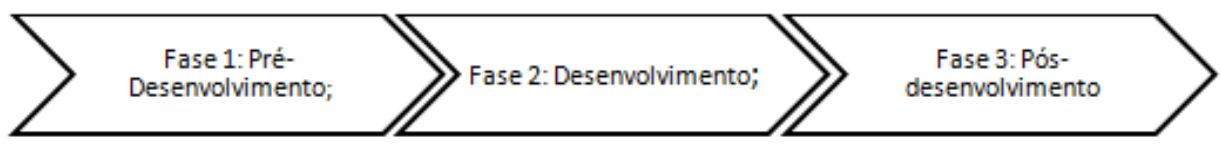

Rozenfeld et al. (2006):

$\left.\left.\left.\left.\sum \begin{array}{c}\text { Fase 1: } \\ \text { Geração do } \\ \text { conceito }\end{array}\right\rangle \begin{array}{c}\text { Fase 2: } \\ \text { Planejamento } \\ \text { do produto }\end{array}\right\rangle \begin{array}{c}\text { Fase 3: } \\ \text { Detalhamento } \\ \text { do produto }\end{array}\right\rangle \begin{array}{c}\text { Fase 4: } \\ \text { Detalhamento } \\ \text { do processo }\end{array}\right\rangle$

Takahashi\& Takahashi (2007):

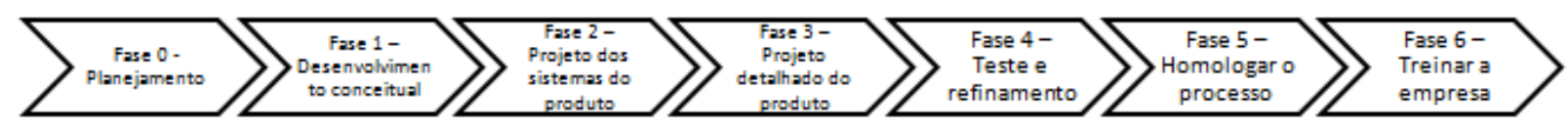

Ulrich e Eppinger (1995)

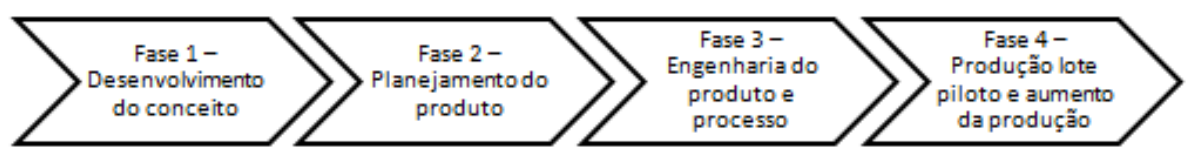

Fonte: Wheelwright e Clark (1992); Cooper (1993); Ulrich e Eppinger (1995); Baxter (2003); Rozenfeld et al. (2006); Takahashi e Takahashi (2007)

Os modelos propostos por Cooper (1993) e Ulrich e Eppinger (1995) compreendem atividades de feedback entre as fases. Essa interação acontece porque as informações geradas em uma fase retroalimentam as fases anteriores. Embora cada uma dessas denominações traga algumas diferenças, em essência são muito semelhantes. Algumas dessas definições trazem maior identidade com a engenharia de produção, como as de Wheelwright e Clark (1992) e Rozenfeld et al. (2006) e outras em Marketing como a de Cooper (1993). De um modo geral não há diferenças significativas nas divisões estabelecidas pelos autores. As maiores diferenças estão no número de fases que varia 
de acordo com cada modelo.

\section{Metodologia de pesquisa}

\subsection{Desenvolvimento do novo produto}

O objetivo principal deste trabalho é detalhar as etapas de uma metodologia específica aplicada no projeto do porta esmalte. O desenvolvimento e a execução do projeto foram baseados em um modelo específico de PDP, formulado com base nos já consagrados pela literatura. Todas as etapas de concepção do produto são detalhadas a seguir.

\subsubsection{Concepção do produto}

\section{a) Geração de ideias}

A geração de idéias consistiu num estudo inicial onde foram identificadas as oportunidades e necessidade do mercado, visando à descoberta de necessidades dos consumidores e a oportunidade de novos produtos. Essa etapa teve como princípio norteador a idealização de um produto que tivesse as seguintes características:

- Utilidade para uma função específica na sociedade em geral;

- Processo de concepção de baixa complexidade;

- Viabilidade econômica;

- Inovador;

- Possibilidade de construção do protótipo;

- Matéria-prima simples.
A partir dos caracteres estabelecidos tornou-se possível apontar um produto do tipo modelo de utilidade que além de auxiliar como ferramenta de trabalho na profissão de manicures em geral, mas especificamente aquelas que trabalham em domicílio, poderá servir para a população feminina como objeto de organização e praticidade. $\mathrm{O}$ produto proposto e idealizado, à priori, foi um porta esmaltes.

\section{b) Definição do problema}

Por meio das características analisadas identificou-se a oportunidade de conceber um produto que pudesse auxiliar na tarefa de organizar, guardar e transportar os esmaltes e demais utensílios utilizados na atividade de fazer as unhas. Essa identificação foi possível por meio da observação direta de profissionais manicures e relatos do público feminino em geral sobre a inexistência de um lugar apropriado para guardar suas ferramentas de fazer as unhas e seus esmaltes.

\section{c) Análise do problema}

Com a descoberta do mercado favorável e da necessidade dos consumidores pelo produto em questão o próximo passo foi à compreensão do problema. Por meio de pesquisas, observação direta e conversas informais com as profissionais foram levantados alguns tópicos importantes envolvidos na tarefa pela a qual o produto esta sendo destinado.

- Os produtos disponíveis e comercializados para a função de guardar/ transportar e 
organizar os esmaltes apresenta-se incompletos e dificilmente realizam todas essas funções simultaneamente;

- Nos produtos similares os lugares para guardar os fracos são desapropriados, impossibilitando sua organização e segurança;

- O armazenamento inapropriado dos frascos ocasiona, em alguns casos, o desperdício do produto e a redução da sua vida útil;

- As manicures necessitam de um lugar prático e organizado para guardar os fracos de modo que o tempo de procura e escolha de um determina do esmaltes seja reduzido tendo em vista a forma como os vidros estão arranjados.

- A importância de agrupar e guardar juntamente com os esmaltes os demais utensílios usados na tarefa de fazer as unhas.

Os critérios mencionados possibilitaram o entendimento de que o produto a ser desenvolvido deverá apresentar um arranjo organizado com economia de espaço para os usuários além de estabelecer a posição correta de armazenamento. Ele também apontou uma melhoria na atividade de transporte, tornando seu uso ou manuseio mais confortável e ergonomicamente correto.

3.1.2 Análise de mercado
$\mathrm{Na}$ análise da viabilidade foi elaborado um estudo por meio de pesquisas externas que trouxessem contribuições significativas no âmbito do projeto do produto. Foram realizadas investigações sobre a existência de produtos do tipo ou similares, dos preços praticados pelo mercado com relação a esses produtos e a posição dos consumidores com relação a esses preços, ou seja, quanto que o mercado estaria disposto a pagar pelo bem que esta sendo ofertado.

\section{b) Especificação de oportunidades}

Essa etapa do projeto buscou indicar as perspectivas do mercado, os aspectos críticos que poderiam afetar positiva ou negativamente, os benefícios trazidos pelo produto, os principais concorrentes e o público-alvo que ele iria atender.

\section{c) Concorrentes}

Como concorrentes têm-se produtos que apresentam uma única funcionalidade, ou seja, que se destinam somente para o armazenamento de esmaltes. A fim de avaliar a viabilidade da idéia, realizou-se uma comparação entre os produtos similares e os principais concorrentes disponíveis no mercado. As informações estão detalhadas no Quadro 1. 
QUADRO 1: ANÁLISE DOS PRODUTOS SIMILARES E CONCORRENTES

\begin{tabular}{|c|c|c|c|}
\hline Item & Desvantagem & Preço & Foto \\
\hline $\begin{array}{c}\mathbf{A} \\
\text { Caixas de } \\
\text { papelão }\end{array}$ & $\begin{array}{l}\text { - A matéria-prima é de baixa qualidade facilmente } \\
\text { deteriorada ou rasgada; } \\
\text { - Dificuldade de manuseio/transporte; } \\
\text { - Pouca organização dos fracos; }\end{array}$ & $\begin{array}{l}\text { Faixa de } \\
\text { preço entre } \\
\mathrm{R} \$ 20,00 \text { a } \\
\mathrm{R} \$ 30,00\end{array}$ & \\
\hline $\begin{array}{c}\text { B } \\
\text { Nécessaire }\end{array}$ & $\begin{array}{l}\text { - Desorganização dos fracos; } \\
\text { - Pouca variabilidade de tamanho; } \\
\text { - Falta de um compartimento apropriado para guardar } \\
\text { as ferramentas }\end{array}$ & $\begin{array}{l}\text { Faixa de } \\
\text { preço entre } \\
\mathrm{R} \$ 30,00 \text { a } \\
\mathrm{R} \$ 60,00\end{array}$ & \\
\hline $\begin{array}{c}\text { C } \\
\text { Caixa de } \\
\text { madeira }\end{array}$ & $\begin{array}{l}\text { - Material sujeito a riscos como quebra; } \\
\text { - Dificuldade de manuseio/transporte; } \\
\text { - Pesada; } \\
\text { - Ocupa muito espaço } \\
\text { - Falta de um compartimento apropriado para guardar } \\
\text { as ferramentas }\end{array}$ & $\begin{array}{l}\text { Faixa de } \\
\text { preço entre } \\
\mathrm{R} \$ 30,00 \text { a } \\
\mathrm{R} \$ 150,00\end{array}$ & \\
\hline
\end{tabular}

Fonte: Autores (2013)

\subsubsection{Planejamento do produto}

\section{a) Projeto informacional}

No projeto informacional foram definidas as especificações meta do projeto. Essas especificações são levadas as próximas fases, norteando todo o processo. Para delimitar o estudo e traçar os requisitos básicos do produto foi utilizada a casa da qualidade ou matriz QFD. Essa ferramenta possibilitou uma análise dos requisitos dos clientes em conformidade com os do produto e com isso pode-se chegar às especificações meta. A casa da qualidade esta ilustrada na Figura 2.

\section{b) Projeto preliminar}

O projeto preliminar possibilitou apresentar o esboço inicial do projeto, bem como sua forma, definição, materiais e componentes. Nessa etapa buscou-se traçar o desenho do arranjo do conjunto e dos componentes, descrevendo o desenho esquemático.

Ainda nessa etapa foram esboçados diversos desenhos, tendo em vista a seleção da melhor proposta em relação à forma, materiais para a produção, usabilidade e fabricação. Por meio dos rascunhos e do detalhamento de cada requisito foi escolhido o esboço com base nos recursos disponíveis para confecção do protótipo. Esse desenho é mostrado na Figura 3. 


\section{ReLAInEF}

FIGURA 2: A CASA DA QUALIDADE
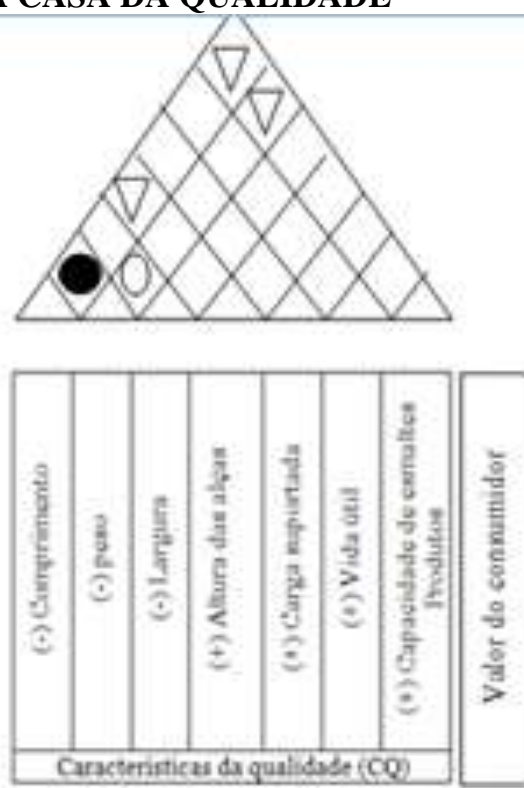

Ancilise do mereado

\begin{tabular}{|c|c|c|}
\hline \multirow{14}{*}{ \& } & \multirow{6}{*}{ Fescicoulidade } & Set leve \\
\hline & & Ser maleavd \\
\hline & & 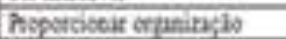 \\
\hline & & Fus ensasos \\
\hline & & Sel fact be pusde \\
\hline & & Finlinpen \\
\hline & \multirow{4}{*}{ Apatescia } & Denfert atuesie \\
\hline & & Duferenties coses \\
\hline & & 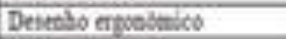 \\
\hline & & Nericalateis \\
\hline & \multirow{4}{*}{ Resisthail } & Set Gariven \\
\hline & & Sert seliterth \\
\hline & & Xis exy \\
\hline & & Xis frigits che $\$$ missiof \\
\hline
\end{tabular}
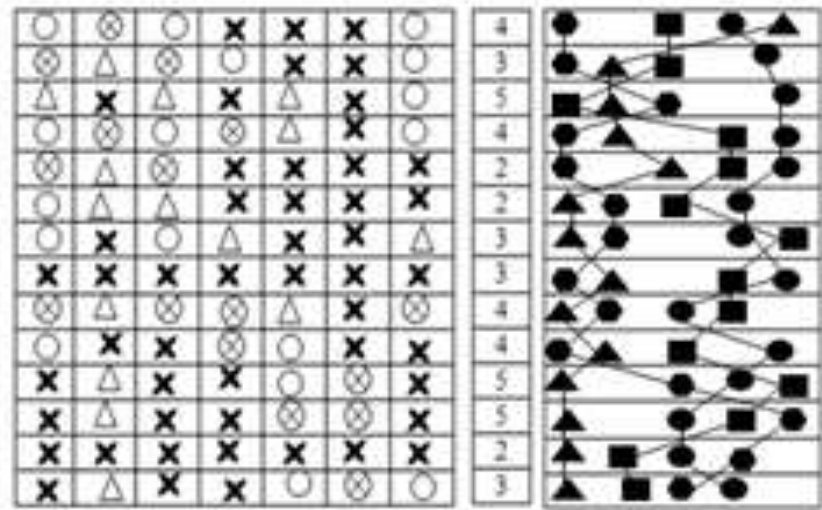

\begin{tabular}{|c|c|c|c|c|c|c|c|c|c|}
\hline \multicolumn{2}{|r|}{ Lerondi } & thaidader & c & $G$ & C & C & $\overline{k e}$ & $A$ & t \\
\hline C & Ceerrananato & Padate A & 15 & 700 & 70 & 6 & 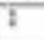 & $\mathrm{t}$ & 20.23 \\
\hline 0 & Crams & & & & & & & & \\
\hline$k_{1}$ & Khogram & Wadate al & 25 & 900 & is & 9 & 3 & 5 & 25. \\
\hline$\frac{A}{0}$ & Anes & hadatio & 90 & $1060^{-1}$ & 30 & 10 & 3 & 3 & 20.30 \\
\hline & & Soene Prodat: & 35 & 300 & 35 & 30 & 5 & 5 & 30.4 \\
\hline
\end{tabular}

\begin{tabular}{|c|c|c|c|c|c|c|c|}
\hline $\begin{array}{l}\text { Vales d } \\
\text { Impontiniás }\end{array}$ & 101 & 64 & 85 & 72 & $\pi$ & 65 & $\overline{10}$ \\
\hline $\begin{array}{c}\text { Cuisticasse } \\
\text { de } \\
\text { ingondeois }\end{array}$ & $t^{2}$ & $x^{\circ}$ & $2^{\circ}$ & $5^{\circ}$ & $F$ & $6^{\prime}$ & $3^{\prime \prime}$ \\
\hline
\end{tabular}

Fonte: Autores (2013) 


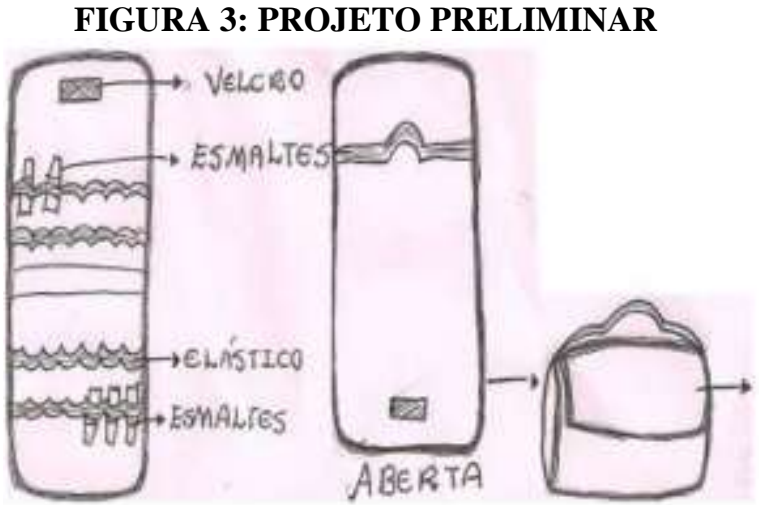

Fonte: Autores (2013)

\section{c) Projeto detalhado e protótipo}

O projeto detalhado proporcionou a definição e análise da forma geométrica do produto. Foi possível realizar algumas correções em relação ao projeto preliminar. Também foi feita a finalização e especificação técnica, bem como a seleção dos materiais, detalhar o produto e planejar os processos de fabricação e montagem. Essa etapa também foi importante porque possibilitou identificar muitas características e detalhes que por vezes fica oculta na etapa de concepção. A Figura 4 é referente ao projeto detalhado do porta esmaltes.

\section{FIGURA 4: PROJETO DETALHADO}

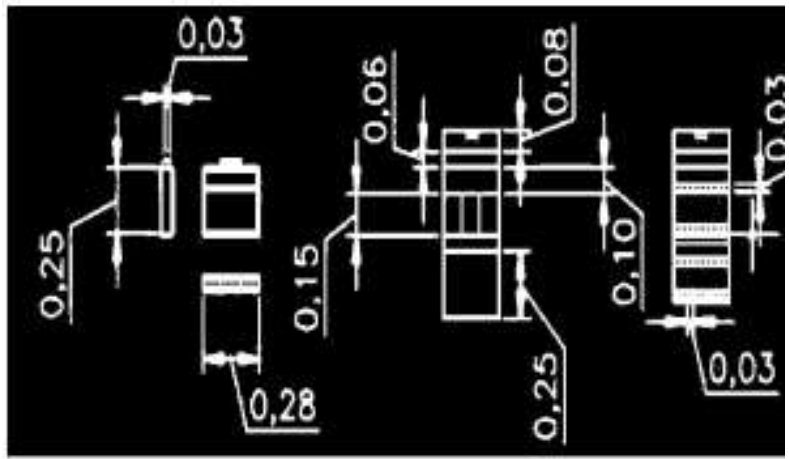

Fonte: Autores (2013)
Nessa etapa identificaram-se dificuldades principalmente para selecionar o material mais adequado para construção do protótipo. Pois o correto seria utilizar como matéria-prima principal o material denominado lona devido a sua qualidade, resistência e dureza. No entanto devido à escassez desse insumo o substituímos por outro (napa) com qualidade inferior. Os demais materiais utilizados para construção do protótipo foram: elástico, velcro, plástico transparente, víeis, cola, zíper e linha.

Após a seleção dos materiais e definição das medidas o processo de fabricação do protótipo foi iniciado. Esse processo foi terceirizado por um profissional da área de corte e costura. $\mathrm{O}$ protótipo físico do produto representa não só o resultado da metodologia desenvolvido como também proporciona a realização de testes de usabilidade e funcionalidade do produto em si. O protótipo funcional do produto proposto é apresentado na Figura 5. Vale ressaltar que ele foi construído nas dimensões reais do produto.

FIGURA 5: PROTÓTIPO

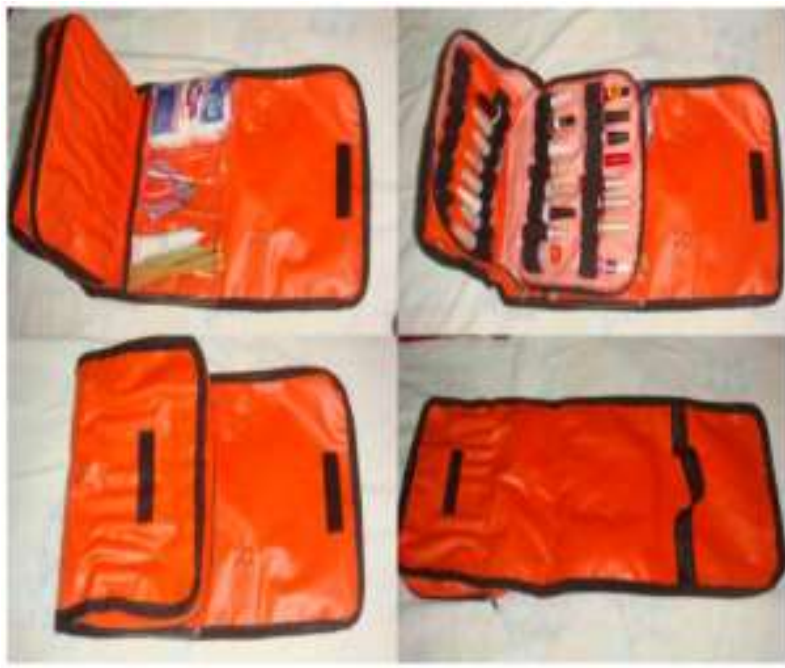

Fonte: Autores (2013) 


\section{d) A ergonomia do produto}

Após a concepção do protótipo chegou-se a etapa de testar e avaliar ergonomicamente o produto. Tomando como base o autor Gomes Filho (2003), a avaliação foi concebida segundo três fatores básicos: Requisito do Projeto, Ações de Manejo e Ações de Percepção. De forma geral, observaram-se alguns quesitos importantes:

- O porta esmalte permite guardar os esmaltes no seu interior na posição vertical, de maneira segura e organizada;

- Sua utilização é simples e seu uso é eficiente;

- Alças ajustáveis a todos os tipos de altura;

- As alças permitem um bom manuseio do produto;

- Exige pouca habilidade e pouca precisão para seu uso;

QUADRO 2: CUSTOS PARA CONSTRUÇÃO DO PROTÓTIPO

\begin{tabular}{cccccc}
\multicolumn{7}{c}{ QUADRO 2: CUSTOS PARA CONSTRUÇÃO DO PROTÓTIPO } \\
\hline Item & $\begin{array}{c}\text { Peça/ } \\
\text { Componente }\end{array}$ & $\begin{array}{c}\text { Unidade } \\
\text { medida }\end{array}$ & $\begin{array}{c}\text { Quantidade } \\
\text { material }\end{array}$ & $\begin{array}{c}\text { Valor } \\
\text { Material } \\
\text { (R\$) }\end{array}$ & $\begin{array}{c}\text { Porcentagem } \\
\text { do custo } \\
\text { total(\%) }\end{array}$ \\
\hline 1 & Napa & $\mathrm{M}$ & 0,5 & 2,50 & 12,5 \\
2 & Elástico & $\mathrm{M}$ & 4 & 4,30 & 21,5 \\
3 & Velcro & $\mathrm{Cm}$ & 10 & 0,50 & 2,5 \\
4 & Plástico & $\mathrm{Cm}$ & 45 & 2,00 & 10 \\
& transparente & $\mathrm{M}$ & 2,5 & 1,40 & 7 \\
5 & Víeis & $\mathrm{G}$ & 5 & 0,30 & 1,5 \\
6 & Cola & $\mathrm{M}$ & 10 & 3,00 & 15 \\
7 & Linha & $\mathrm{Cm}$ & 60 & 2,00 & 10 \\
8 & Zíper & Unidade & - & 5,00 & 25 \\
9 & Mão -de -obra & & & $\mathbf{2 0 , 0 0}$ & $\mathbf{1 0 0}$ \\
Total & & & & & \\
\hline
\end{tabular}

Fonte: Autores (2013)
- Transporte confortável e seguro

3.1.4 Detalhamento da produção

\section{a) Custo do produto}

Nessa etapa buscou-se fazer um estudo mais aprofundado dos materiais a serem utilizados para a fabricação, a fim de mensurar os custos e definir o processo.

O Quadro 2 apresenta apenas uma estimativa de custos com materiais para a produção de uma unidade do Porta esmalte, a partir do projeto detalhado. Para avaliar o custo do produto, também é necessário avaliar os custos de produção, considerando mão-de-obra, energia e outros insumos. O que não foi possível realizar, de forma detalhada, considerando o contexto deste projeto. 


\section{ReLAInEF}

- $\quad$ Processo de produção

Para avaliar a viabilidade técnica e econômica de um novo produto é necessário projetar o processo de produção. A Figura 6 ilustra o fluxograma do processo de produção.

FIGURA 6: FLUXOGRAMA DO PROCESSO DE PRODUÇÃO

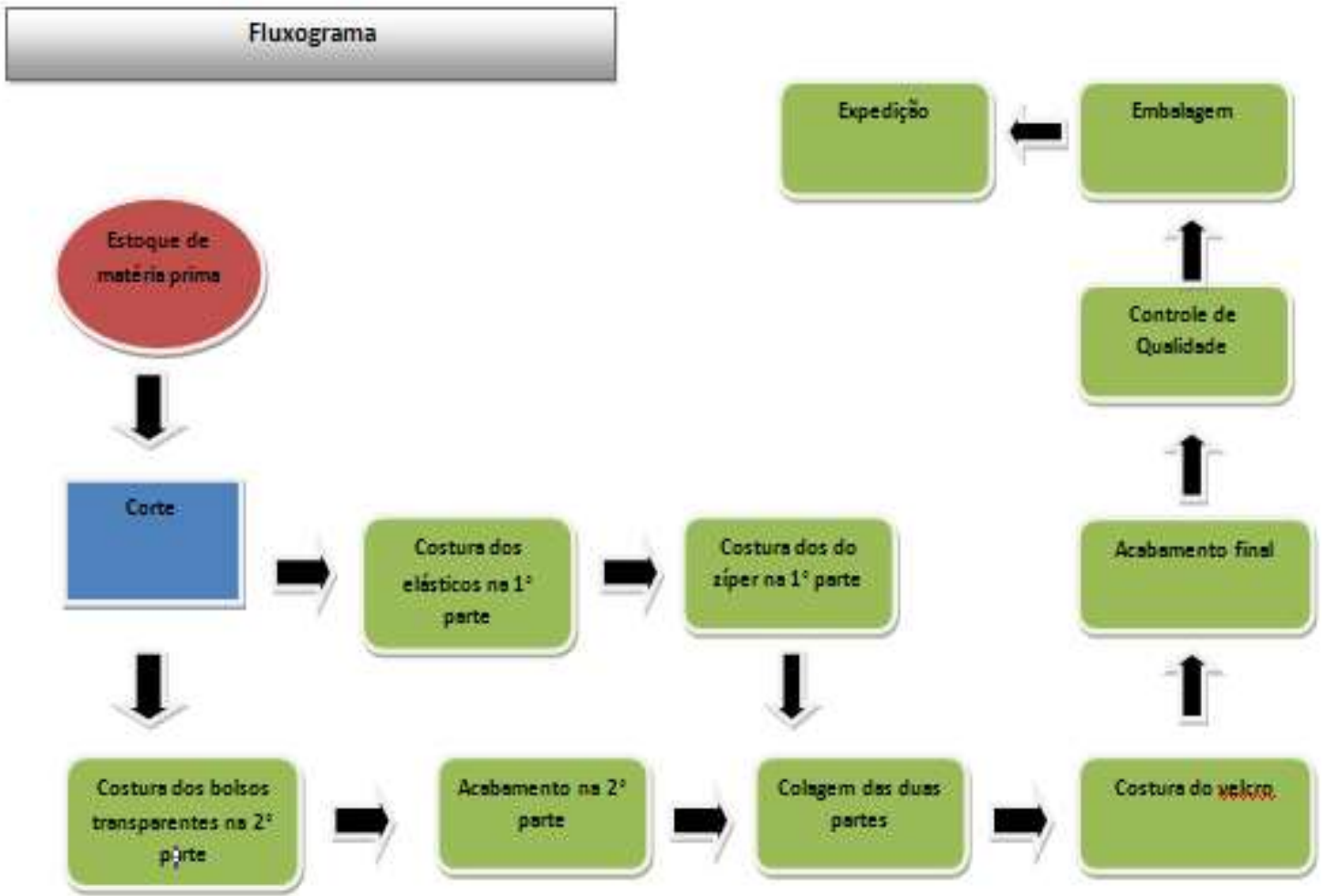

Fonte: Autores (2013)

\section{Conclusões}

A atividade de projetar e desenvolver novos produtos esta intimamente relacionada a escolha de um método. E essa escolha esta condicionada e relacionada ao tipo de produto, ao seu processo de produção, sua complexidade e, principalmente, com as exigências do mercado consumidor. É importante mencionar que o modelo tende a atender as necessidades da equipe multidisciplinar para que o produto seja visto por vários âmbitos.
O resultado do método desenvolvido foi um produto denominado "porta esmalte", composto com base em uma metodologia específica, elaborada por meio das já existentes e consistentes na literatura. $\mathrm{O}$ produto proposto é útil e de pouca complexidade, que supri as necessidades latentes ainda não supridas pelos produtos já existentes.

A adoção de um método também contribuiu de forma significativa no gerenciamento das fases do processo, reforçando a concepção de que a qualidade de um projeto não depende unicamente da complexidade do objeto e que 


\section{ReLAInEF}

idéias simples e inovadoras fluem em diferentes momentos e lugares.

A construção do protótipo serviu para evidenciar a possibilidade de sucesso que o produto poderia ter devido a sua viabilidade. No entanto, é importante ressaltar que para transformar a ideia em um negócio faz-se necessário um estudo aprofundado sobre questões técnicas, econômicas e comerciais, além da execução de um plano de negócio a fim de diminuir os riscos e incertezas advindos do mercado.

\section{Referências}

BAXTER, M. Projeto de Produto: Guia prático para o desenvolvimento de novos produtos. $2^{\text {a }}$ Ed. São Paulo: Edgard Blucher, 2003.

CHASE, R. B.; JACOBS, F. R.; AQUILANO, N. J. Administração da produção e operações para vantagens competitivas. $11^{\mathrm{a}}$ ed. São Paulo: McGraw-Hill, 2006.

COOPER, R. G. Winning at new products: accelerating the process form idea to launch. Reading, Perseus Books, 1993.

GOMES FILHO, J. Sistema Técnico de Leitura Ergonômica. São Paulo: Escrituras, 2003.

KAMINSKI, P.C. Desenvolvimento de produtos com planejamento, criatividade e qualidade. CTC: Rio de Janeiro, 2000.

KOTLER, P. Administração de marketing. 10 ed. Pretice Hall: São Paulo, 2000.

MACHADO, Marcio Cardoso; TOLEDO, Nilton Nunes. Gestão do processo e desenvolvimento de produto: uma abordagem baseada na criação de valor. São Paulo: Atlas, 2008.

ROZENFELD, H.; FORCELLINI, F.A.; AMARAL, D.C.; TOLEDO, J.C.; SILVA, S.L.;
ALLIPRANDINI, D.H.; SCALICE， R.K. Gestão de desenvolvimento de produtos: uma referência para a melhoria do processo. São Paulo: Saraiva, 2006.

ROMEIRO FILHO, E.; FERREIRA, C.V.; CAUCHICK MIGUEL, P. A.; GOUVINHAS, R. P.; NAVEIRO, R. M.. Projeto do Produto. $1^{a}$ ed. São Paulo: Campus, 2009.

TAKAHASHI, S. \& TAKAHASHI, V. Gestão de inovação de produtos: estratégia, processo, organização e conhecimento. Rio de Janeiro: Editora Campus, 2007.

ULRICH, K.; EPPINGER, S. Product design and development. New York: McGraw-Hill, 1995

WHEELWRIGHT, S. CLARK, K. B.; Revolutionizing product development: Quantum Leaps in Speed, Efficiency, and Quality. New York, The Free Press, 1992. 